\title{
EXISTENCE OF SOLUTIONS FOR GENERALIZED NONLINEAR MIXED VARIATIONAL-LIKE INEQUALITIES IN BANACH SPACES
}

\author{
YALI ZHAO, ZUNQUAN XIA, ZEQING LIU, AND SHIN MIN KANG
}

Received 26 April 2005; Revised 5 December 2005; Accepted 18 December 2005

We introduce and study a new class of generalized nonlinear mixed variational-like inequalities in reflexive Banach spaces. By applying Ding's technique we prove several existence and uniqueness theorems of solutions for the generalized nonlinear mixed variational-like inequality, extend the auxiliary problem technique to suggest and analyze an iterative method to compute the approximate solutions of the generalized nonlinear mixed variational-like inequality, and establish the convergence criteria of the iterative method. The results presented in this paper improve, extend, and unify many known results in this area.

Copyright (c) 2006 Hindawi Publishing Corporation. All rights reserved.

\section{Introduction}

Variational inequality theory has appeared as an effective and powerful tool to study and investigate a wide class of problems arising in pure and applied sciences including elasticity, optimization, economics, transportation, and structural analysis, see, for example, $[1-8,10-37]$ and the references therein. The classical variational inequality problem has been generalized in various directions for its own development and its applications. Mixed variational-like inequality is an important and useful generalization of the classical variational inequality. On the other hand, constructing effective numerical algorithm is an important and difficult problem in the variational inequality theory. Cohen [3] first extended the auxiliary variational inequality technique to suggest and analyze an innovative iterative method for solutions of mixed variational inequalities in reflexive Banach spaces. Ding [8] proved an existence-uniqueness theorem of solutions of a new class of nonlinear mixed variational-like inequalities in reflexive Banach spaces by making use of minimax inequality due to Ding and Tan [9]. Following the idea of Cohen [3], Ding suggested and analyzed a quite general iterative method to compute the approximate solutions and proved the convergence of the iterative method.

Motivated and inspired by the research work going on in this field, we will introduce and study a new class of generalized nonlinear mixed variational-like inequalities in reflexive Banach spaces, which includes many variational inequalities and variational-like 
2 Generalized nonlinear mixed variational-like inequalities

inequalities as special cases, for details, see [1-8, 10-37] and the references therein. Following the idea of Ding [8], we prove several existence and uniqueness theorems of solutions for the generalized nonlinear mixed variational-like inequality, extend the auxiliary problem technique to suggest and analyze an iterative method to compute the approximate solutions of the generalized nonlinear mixed variational-like inequality, and obtain the convergence criteria of the iterative method. The results presented in this paper improve, extend, and unify many known results in this area.

\section{Preliminaries}

Let $D$ be a nonempty convex subset of a Banach space $E$ with dual $E^{*}$ and let $\langle u, v\rangle$ be the dual pairing between $u \in E^{*}$ and $v \in E$. Let $T, A, B, C: D \rightarrow E^{*}, M, N: E^{*} \times E^{*} \rightarrow E^{*}$, and $\eta: D \times D \rightarrow E$ be mappings. Let $b: D \times D \rightarrow R$ be a real-valued functional. Now we consider the following generalized nonlinear mixed variational-like inequality problem. For a given $w^{*} \in E^{*}$, find $u \in D$ such that

$$
\left\langle N(T u, A u)-M(B u, C u)+w^{*}, \eta(v, u)\right\rangle+b(u, v)-b(u, u) \geq 0, \quad \forall v \in D,
$$

where the functional $b$ is nondifferential and satisfies the following conditions:

(c1) $b$ is linear in the first argument;

(c2) for each $u \in D, b(u, \cdot)$ is a convex and lower semicontinuous functional on $D$;

(c3) $b$ is bounded, that is, there exists a constant $v>0$ such that

$$
b(u, v) \leq v\|u\|\|v\|, \quad \forall u, v \in D
$$

(c4) for all $u, v, w \in D, b(u, v)-b(u, w) \leq b(u, v-w)$.

Special cases. (A) If $M(B u, C u)=0$ for all $u \in D$, and $-w^{*}$ is given, then the problem (2.1) reduces to the following variational-like inequality problem: for a given $w^{*} \in E^{*}$, find $u \in D$ such that

$$
\left\langle N(T u, A u)-w^{*}, \eta(v, u)\right\rangle+b(u, v)-b(u, u) \geq 0, \quad \forall v \in D,
$$

which was introduced and studied by Ding [8].

(B) If $M(B u, C u)=M(B u)$ for all $u \in D$, then the problem (2.1) is equivalent to the following problem: for a given $w^{*} \in E^{*}$, find $u \in D$ such that

$$
\left\langle N(T u, A u)-M(B u)+w^{*}, \eta(v, u)\right\rangle+b(u, v)-b(u, u) \geq 0, \quad \forall v \in D .
$$

(C) If $M(B u)=w^{*}$ for all $u \in D$, then the problem (2.4) is equivalent to the following problem: find $u \in D$ such that

$$
\langle N(T u, A u), \eta(v, u)\rangle+b(u, v)-b(u, u) \geq 0, \quad \forall v \in D,
$$

which was introduced and considered by Liu and Tang [26].

(D) If $\eta(v, u)=v-u, N(T u, A u)=T u-w^{*}$, and $b(u, v)=f(v)$ for all $u, v \in D$, where $f: D \rightarrow(-\infty,+\infty)$, then the problem (2.5) is equivalent to the following problem: find 
$u \in D$ such that

$$
\left\langle T u-w^{*}, v-u\right\rangle+f(v)-f(u) \geq 0, \quad \forall v \in D,
$$

which is originally considered by Cohen [3].

For the appropriate and suitable choices of mappings $M, N, \eta, b, T, A, B, C, D, w^{*}$, and the space $E$, we can obtain many known and new classes of variational inequalities and variational-like inequalities as special cases of the problem $(2.1)$, see $[1-4,6,7,16-19,25]$ and the references therein. In brief, the problem (2.1) is more general and a unifying one, which is also one of the main motivations of this paper.

Now, we recall the following definitions and some known results.

Definition 2.1. Let $D$ be a nonempty subset of a Banach space $E$ with the dual space $E^{*}$. Let $T: D \rightarrow E^{*}$ and $\eta: D \times D \rightarrow E$ be two mappings. The mapping $T$ is called

(1) $\eta$-strongly monotone if there exists a constant $\alpha>0$ such that

$$
\langle T u-T v, \eta(u, v)\rangle \geq \alpha\|u-v\|^{2}, \quad \forall u, v \in D
$$

(2) $\eta$-relaxed Lipschitz if there exists a constant $\alpha \geq 0$ such that

$$
\langle T u-T v, \eta(u, v)\rangle \leq-\alpha\|u-v\|^{2}, \quad \forall u, v \in D ;
$$

(3) Lipschitz continuous if there exists a constant $L>0$ such that

$$
\|T u-T v\| \leq L\|u-v\|, \quad \forall u, v \in D
$$

(4) $\eta$-generalized pseudocontractive if there exists a constant $\alpha>0$ such that

$$
\langle T u-T v, \eta(u, v)\rangle \leq \alpha\|u-v\|^{2}, \quad \forall u, v \in D
$$

(5) $\eta$-relaxed monotone if there exists a constant $\alpha>0$ such that

$$
\langle T u-T v, \eta(u, v)\rangle \geq-\alpha\|u-v\|^{2}, \quad \forall u, v \in D .
$$

Definition 2.2. Let $D$ be a nonempty subset of a Banach space $E$ with the dual space $E^{*}$, let $T, A: D \rightarrow E^{*}, N: E^{*} \times E^{*} \rightarrow E^{*}$, and $\eta: D \times D \rightarrow E$ be mappings, let $N(T, A)(u)=$ $N(T u, A u)$.

(1) $N$ is said to be Lipschitz continuous in the first argument if there exists a constant $\alpha>0$ such that

$$
\|N(u, w)-N(v, w)\| \leq \alpha\|u-v\|, \quad \forall u, v, w \in E^{*} .
$$

(2) $N$ is said to be $\eta$-cocoercive in the first argument with respect to $T$ if there exists a constant $\alpha>0$ such that

$$
\langle N(T u, w)-N(T v, w), \eta(u, v)\rangle \geq \alpha\|T u-T v\|^{2}, \quad \forall u, v \in D, w \in E^{*}
$$


4 Generalized nonlinear mixed variational-like inequalities

(3) $N$ is said to be $\eta$-strongly monotone in the first argument with respect to $A$ if there exists a constant $\alpha>0$ such that

$$
\langle N(A u, w)-N(A v, w), \eta(u, v)\rangle \geq \alpha\|u-v\|^{2}, \quad \forall u, v \in D, w \in E^{*} .
$$

(4) $N$ is said to be $\eta$-relaxed monotone in the first argument with respect to $A$ if there exists a constant $\alpha>0$ such that

$$
\langle N(A u, w)-N(A v, w), \eta(u, v)\rangle \geq-\alpha\|u-v\|^{2}, \quad \forall u, v \in D, w \in E^{*} .
$$

(5) $N$ is said to be Lipschitz continuous in the first argument with respect to $A$ if there exists a constant $\alpha>0$ such that

$$
\|N(A u, w)-N(A v, w)\| \leq \alpha\|u-v\|, \quad \forall u, v \in D, w \in E^{*} .
$$

(6) $\eta$ is said to be Lipschitz continuous if there exists a constant $\delta \geq 0$ such that

$$
\|\eta(u, v)\| \leq \delta\|u-v\|, \quad \forall u, v \in D .
$$

Similarly, the Lipschitz continuity, $\eta$-strong monotonicity, relaxed monotonicity, cocoercivity, and generalized pseudocontractivity of $N$ can be defined in the second argument.

(7) $N(T u, A u)$ is said to be $\eta$-strongly monotone, if there exists a constant $\alpha>0$ such that

$$
\langle N(T u, A u)-N(T w, A w), \eta(u, w)\rangle \geq \alpha\|u-w\|^{2}, \quad \forall u, w \in D .
$$

(8) $N(T u, A u)$ is said to be $\eta$-generalized pseudocontractive if there exists a constant $\alpha>0$ such that

$$
\langle N(T u, A u)-N(T w, A w), \eta(u, w)\rangle \leq \alpha\|u-w\|^{2}, \quad \forall u, w \in D .
$$

The following notion is a generalization of the corresponding notion introduced by Ding and Tarafdar [10].

Definition 2.3. Let $D$ be a nonempty convex subset of a Banach space $E$. Let $T: D \rightarrow E^{*}$ and $\eta: D \times D \rightarrow E$ be mappings. $T$ and $\eta$ are said to have 0 -diagonally concave relation with respect to $w^{*} \in E^{*}$, if the function $\varphi: D \times D \rightarrow(-\infty,+\infty]$ defined by

$$
\varphi(u, v)=\left\langle T u+w^{*}, \eta(u, v)\right\rangle
$$

is 0 -diagonally concave in $v$, that is, for any finite set $\left\{v_{1}, \ldots, v_{m}\right\} \subset D$ and for any $u=$ $\sum_{i=1}^{m} \lambda_{i} v_{i}$ with $\lambda_{i} \geq 0$ and $\sum_{i=1}^{m} \lambda_{i}=1, \sum_{i=1}^{m} \lambda_{i} \varphi\left(u, v_{i}\right) \leq 0$.

Remark 2.4. Let $D$ be a nonempty convex subset of a Banach space $E$ with the dual space $E^{*}$. Let $T: D \rightarrow E^{*}$ and $\eta: D \times D \rightarrow E$ be mappings such that $\eta(u, u)=0$ for all $u \in D$. Suppose that for given $u \in D$ and $w^{*} \in E^{*}$, the functional $v \mapsto\left\langle T u+w^{*}, \eta(u, v)\right\rangle$ is concave. Then $T$ and $\eta$ have the 0 -diagonally concave relation with respect to $w^{*}$.

Lemma 2.5 [8]. Let $D$ be a nonempty convex subset of a topological vector space and let $\varphi: D \times D \rightarrow[-\infty,+\infty]$ be such that 
(a) for each $x \in D, y \mapsto \varphi(x, y)$ is lower semicontinuous on each nonempty compact subset of $D$,

(b) for each nonempty finite set $\left\{x_{1}, \ldots, x_{m}\right\} \subset D$ and for each $y=\sum_{i=1}^{m} \lambda_{i} x_{i}$ with $\lambda_{i} \geq 0$ and $\sum_{i=1}^{m} \lambda_{i}=1, \min _{1 \leq i \leq m} \varphi\left(x_{i}, y\right) \leq 0$,

(c) there exist a nonempty compact convex subset $X_{0}$ of $D$ and a nonempty compact subset $K$ of $D$ such that for each $y \in D \backslash K$, there is an $x \in \operatorname{co}\left(X_{0} \cup\{y\}\right)$ with $\varphi(x, y)>0$.

Then there exists a $\tilde{y} \in K$ such that $\varphi(x, \tilde{y}) \leq 0$ for all $x \in D$.

\section{Existence and uniqueness theorems}

We now prove the following results.

THeOREM 3.1. Let $D$ be a nonempty convex subset of a reflexive Banach space $E$ with dual space $E^{*}$. Let $T, A, B, C: D \rightarrow E^{*}, M, N: E^{*} \times E^{*} \rightarrow E^{*}, \eta: D \times D \rightarrow E$ be mappings, let $w^{*} \in E^{*}$ be given, and let $b: D \times D \rightarrow(-\infty,+\infty]$ be a functional such that

(a) the mapping $u \mapsto N(T u, A u)$ is continuous from the weak topology on $E$ to the strong topology on $E^{*}$ such that $N$ is $\eta$-strongly monotone in the first argument with respect to $T$ with constant $\alpha>0$, and is $\eta$-relaxed monotone in the second argument with respect to $A$ with constant $\lambda>0$,

(b) the mapping $u \mapsto M(B u, C u)$ is continuous from the weak topology on $E$ to the strong topology on $E^{*}$ such that $M$ is $\eta$-generalized pseudocontractive in the first argument with respect to $B$ with constant $\beta>0$, and is $\eta$-relaxed Lipschitz in the second argument with respect to $C$ with constant $\xi>0$,

(c) $\eta$ is Lipschitz continuous with constant $\delta \geq 0$ and for each $v \in D, u \mapsto \eta(u, v)$ is continuous from the weak topology to the weak topology and $\eta(v, u)=-\eta(u, v)$ for all $u, v \in D$,

(d) the mapping $u \mapsto N(T u, A u)-M(B u, C u)$ and $\eta$ have the 0-diagonally concave relation with respect to $w^{*}$

(e) $b$ satisfies conditions (c1)-(c4) with $\nu+\lambda+\beta<\alpha+\xi$.

Then the generalized nonlinear mixed variational-like inequality (2.1) has a unique solution $\hat{u} \in D$.

Proof. We first prove that for each fixed $u^{*} \in D$, there exists a unique $\widehat{w} \in D$ such that

$$
\left\langle N(T \widehat{w}, A \widehat{w})-M(B \widehat{w}, C \widehat{w})+w^{*}, \eta(v, \widehat{w})\right\rangle+b\left(u^{*}, v\right)-b\left(u^{*}, \widehat{w}\right) \geq 0, \quad \forall v \in D .
$$

For any fixed $u^{*} \in D$, define a functional $\varphi: D \times D \rightarrow(-\infty,+\infty]$ by

$$
\varphi(v, w)=\left\langle N(T w, A w)-M(B w, C w)+w^{*}, \eta(w, v)\right\rangle+b\left(u^{*}, w\right)-b\left(u^{*}, v\right), \quad \forall v, w \in D
$$

Since $b$ satisfies the conditions (c3) and (c4), it is easy to see that $b$ also satisfies

$$
|b(u, v)-b(u, w)| \leq v\|u\|\|v-w\|, \quad \forall u, v, w \in D,
$$

and hence for each fixed $u \in D, b(u, \cdot)$ is continuous on $D$. Notice that the mappings $w \mapsto$ $N(T w, A w)$ and $w \mapsto M(B w, C w)$ are continuous from the weak topology to the strong 
6 Generalized nonlinear mixed variational-like inequalities

topology. For each $w \in D$ and any sequence $\left\{w_{n}\right\} \subset D$ with $w_{n} \rightarrow w$ in the weak topology, we have $\lim _{n \rightarrow \infty} N\left(T w_{n}, A w_{n}\right)=N(T w, A w), \lim _{n \rightarrow \infty} M\left(B w_{n}, C w_{n}\right)=M(B w, C w)$, and $\eta\left(w_{n}, v\right) \rightarrow \eta(w, v)$ as $n \rightarrow \infty$ in the weak topology and the sequence $\left\{\eta\left(w_{n}, v\right)\right\}$ is bounded. It follows that for each $v \in D$,

$$
\begin{aligned}
& \mid\left\langle N\left(T w_{n}, A w_{n}\right)-M\left(B w_{n}, C w_{n}\right)+w^{*}, \eta\left(w_{n}, v\right)\right\rangle \\
& \quad-\left\langle N(T w, A w)-M(B w, C w)+w^{*}, \eta(w, v)\right\rangle \mid \\
& \leq\left|\left\langle N\left(T w_{n}, A w_{n}\right)-N(T w, A w), \eta\left(w_{n}, v\right)\right\rangle\right| \\
& \quad+\left|\left\langle M\left(B w_{n}, C w_{n}\right)-M(B w, C w), \eta\left(w_{n}, v\right)\right\rangle\right| \\
& \quad+\left|N(T w, A w)-M(B w, C w)+w^{*}, \eta\left(w_{n}, v\right)-\eta(w, v)\right\rangle \mid \\
& \leq|| N\left(T w_{n}, A w_{n}\right)-N(T w, A w)|||| \eta\left(w_{n}, v\right) \| \\
&+|| M\left(B w_{n}, C w_{n}\right)-M(B w, C w)||\left\|\eta\left(w_{n}, v\right)\right\| \\
&+\left|\left\langle N(T w, A w)-M(B w, C w)+w^{*}, \eta\left(w_{n}, v\right)-\eta(w, v)\right\rangle\right| \longrightarrow 0 \quad \text { as } n \rightarrow \infty .
\end{aligned}
$$

It follows that for each $v \in D$, the functional $w \mapsto\left\langle N(T w, A w)-M(B w, C w)+w^{*}\right.$, $\eta(w, v)\rangle$ is weakly continuous on $D$. Therefore, $w \mapsto \varphi(v, w)$ is weakly lower semicontinuous on $D$. We claim that $\varphi$ satisfies the condition (b) of Lemma 2.5. If it is false, then there exists a finite set $\left\{v_{1}, \ldots, v_{m}\right\} \subset D$ and $w=\sum_{i=1}^{m} \lambda_{i} v_{i}$ with $\lambda_{i} \geq 0, \sum_{i=1}^{m} \lambda_{i}=1$, such that $\varphi\left(v_{i}, w\right)>0$ for all $i=1, \ldots, m$, that is,

$$
\left\langle N(T w, A w)-M(B w, C w)+w^{*}, \eta\left(w, v_{i}\right)\right\rangle+b\left(u^{*}, w\right)-b\left(u^{*}, v_{i}\right)>0, \quad i=1, \ldots, m
$$

It follows that

$$
\sum_{i=1}^{m} \lambda_{i}\left\langle N(T w, A w)-M(B w, C w)+w^{*}, \eta\left(w, v_{i}\right)\right\rangle+b\left(u^{*}, w\right)-\sum_{i=1}^{m} b\left(u^{*}, v_{i}\right)>0 .
$$

Note that $b(u, v)$ is convex in the second argument. It follows that

$$
\sum_{i=1}^{m} \lambda_{i}\left\langle N(T w, A w)-M(B w, C w)+w^{*}, \eta\left(w, v_{i}\right)\right\rangle>0
$$

which contradicts the condition (d) of Theorem 3.1. Therefore the condition (b) of Lemma 2.5 holds. Let

$$
\begin{gathered}
r=\frac{1}{\alpha-\lambda-\beta+\xi}\left[\delta\left\|N\left(T u^{*}, A u^{*}\right)-M\left(B u^{*}, C u^{*}\right)\right\|+\delta\left\|w^{*}\right\|+\nu\left\|u^{*}\right\|\right], \\
K=\left\{w \in D:\left\|w-u^{*}\right\| \leq r\right\} .
\end{gathered}
$$


Then $K$ and $X_{0}=\left\{u^{*}\right\}$ are both weakly compact convex subsets of $D$. By the assumptions (a)-(d), for each $w \in D \backslash K$, there exists a $u^{*} \in \operatorname{co}\left(X_{0} \cup\{w\}\right)$ such that

$$
\begin{aligned}
\varphi\left(u^{*}, w\right)= & \left\langle N(T w, A w)-M(B w, C w)+w^{*}, \eta\left(w, u^{*}\right)\right\rangle+b\left(u^{*}, w\right)-b\left(u^{*}, u^{*}\right) \\
= & \left\langle N(T w, A w)-N\left(T u^{*}, A w\right), \eta\left(w, u^{*}\right)\right\rangle \\
& +\left\langle N\left(T u^{*}, A w\right)-N\left(T u^{*}, A u^{*}\right), \eta\left(w, u^{*}\right)\right\rangle \\
& -\left\langle M(B w, C w)-M\left(B u^{*}, C w\right), \eta\left(w, u^{*}\right)\right\rangle \\
& -\left\langle M\left(B u^{*}, C w\right)-M\left(B u^{*}, C u^{*}\right), \eta\left(w, u^{*}\right)\right\rangle \\
& +\left\langle N\left(T u^{*}, A u^{*}\right)-M\left(B u^{*}, C u^{*}\right)+w^{*}, \eta\left(w, u^{*}\right)\right\rangle \\
& +b\left(u^{*}, w\right)-b\left(u^{*}, u^{*}\right) \\
\geq & \left\|w-u^{*}\right\|\left[(\alpha-\lambda-\beta+\xi)\left\|w-u^{*}\right\|-\delta\left\|w^{*}\right\|-\delta \|\right. \\
& \left.\quad N\left(T u^{*}, A u^{*}\right)-M\left(B u^{*}, C u^{*}\right)\|-v\| u^{*} \|\right]>0 .
\end{aligned}
$$

Hence the condition (c) of Lemma 2.5 is also satisfied. By Lemma 2.5, there exists a $\widehat{w} \in K$ such that $\varphi(v, \widehat{w}) \leq 0$ for all $v \in D$, that is,

$$
\left\langle N(T \widehat{w}, A \widehat{w})-M(B \widehat{w}, C \widehat{w})+w^{*}, \eta(\widehat{w}, v)\right\rangle+b\left(u^{*}, \widehat{w}\right)-b\left(u^{*}, v\right) \leq 0, \quad \forall v \in D .
$$

Since $\eta(\widehat{w}, v)=-\eta(v, \widehat{w})$, we see that

$$
\left\langle N(T \widehat{w}, A \widehat{w})-M(B \widehat{w}, C \widehat{w})+w^{*}, \eta(v, \widehat{w})\right\rangle+b\left(u^{*}, v\right)-b\left(u^{*}, \widehat{w}\right) \geq 0, \quad \forall v \in D
$$

Now we prove that $\widehat{w}$ is a unique solution of the problem (3.1). Suppose that $w_{1}, w_{2}$ are arbitrary two solutions of the problem (3.1). It follows that for all $v \in D$,

$$
\begin{aligned}
& \left\langle N\left(T w_{1}, A w_{1}\right)-M\left(B w_{1}, C w_{1}\right)+w^{*}, \eta\left(v, w_{1}\right)\right\rangle+b\left(u^{*}, v\right)-b\left(u^{*}, w_{1}\right) \geq 0, \\
& \left\langle N\left(T w_{2}, A w_{2}\right)-M\left(B w_{2}, C w_{2}\right)+w^{*}, \eta\left(v, w_{2}\right)\right\rangle+b\left(u^{*}, v\right)-b\left(u^{*}, w_{2}\right) \geq 0 .
\end{aligned}
$$

Taking $v=w_{2}$ in (3.12) and $v=w_{1}$ in (3.13), and adding these inequalities, we obtain that

$$
\left\langle N\left(T w_{1}, A w_{1}\right)-M\left(B w_{1}, C w_{1}\right)-N\left(T w_{2}, A w_{2}\right)+M\left(B w_{2}, C w_{2}\right), \eta\left(w_{2}, w_{1}\right)\right\rangle \geq 0 .
$$

By the $\eta$-strong monotonicity of $N$ in the first argument and $\eta$-relaxed strong monotonicity of $N$ in the second argument, the $\eta$-generalized pseudocontractivity of $M$ in the first argument and $\eta$-relaxed Lipschitz continuity of $M$ in the second argument, we get that

$$
(\alpha-\lambda-\beta+\xi)\left\|w_{1}-w_{2}\right\|^{2} \leq 0
$$


Since $\beta+\lambda+\nu<\alpha+\xi$, it follows that $w_{1}=w_{2}$ and $\widehat{w}$ is the unique solution of the problem (3.1). Thus we have proved that for each $u \in D$, there exists a unique solution $w(u)$ satisfying (3.1). Define a mapping $F: D \rightarrow D$ by $u \mapsto w(u)=F u$. We prove that the mapping $F$ is a contraction mapping. Indeed, for any $u_{1}, u_{2} \in D$, there exist $w_{1}=F\left(u_{1}\right), w_{2}=F\left(u_{2}\right)$ such that for all $v \in D$,

$$
\begin{aligned}
& \left\langle N\left(T w_{1}, A w_{1}\right)-M\left(B w_{1}, C w_{1}\right)+w^{*}, \eta\left(v, w_{1}\right)\right\rangle+b\left(u_{1}, v\right)-b\left(u_{1}, w_{1}\right) \geq 0, \\
& \left\langle N\left(T w_{2}, A w_{2}\right)-M\left(B w_{2}, C w_{2}\right)+w^{*}, \eta\left(v, w_{2}\right)\right\rangle+b\left(u_{2}, v\right)-b\left(u_{2}, w_{2}\right) \geq 0 .
\end{aligned}
$$

Taking $v=w_{2}$ in (3.16) and $v=w_{1}$ in (3.17) and adding these inequalities, and considering the assumption on $b$ and $\eta$, we have

$$
\left\|w_{1}-w_{2}\right\| \leq \frac{v}{\alpha-\beta-\lambda+\xi}\left\|u_{1}-u_{2}\right\|
$$

which implies that $F$ is a contraction mapping. Hence there exists a unique point $\hat{u} \in D$ such that $\hat{u}=F(\hat{u})$, that is,

$$
\left\langle N(T \hat{u}, A \hat{u})-M(B \hat{u}, C \hat{u})+w^{*}, \eta(v, \widehat{u})\right\rangle+b(\hat{u}, v)-b(\hat{u}, \hat{u}) \geq 0, \quad \forall v \in D,
$$

and $\hat{u} \in D$ is the unique solution of the generalized nonlinear mixed variational-like inequality problem (2.1). This completes the proof.

As in the proof of Theorem 3.1, we have the following result.

Theorem 3.2. Let $D, E, E^{*}, \eta, w^{*}$ be as in Theorem 3.1. Let $T, A, B, C: D \rightarrow E^{*}, M, N$ : $E^{*} \times E^{*} \rightarrow E^{*}$ be mappings, and let $b: D \times D \rightarrow(-\infty,+\infty]$ be a functional such that

(a) the mapping $u \mapsto N(T u, A u)$ is continuous from the weak topology on $E$ to the strong topology on $E^{*}$ and $\eta$-strongly monotone with constant $\alpha_{1}>0$,

(b) the mapping $u \rightarrow M(B u, C u)$ is continuous from the weak topology on $E$ to the strong topology on $E^{*}$ and $\eta$-generalized pseudocontractive with constant $\alpha_{2}>0$,

(c) $\eta$ satisfies the condition (c) of Theorem 3.1, and the condition (d) of Theorem 3.1 still holds,

(d) b satisfies conditions (c1)-(c4) with $\nu+\alpha_{2}<\alpha_{1}$.

Then the generalized nonlinear mixed variational-like inequality (2.1) has a unique solution $\bar{u} \in D$.

Remark 3.3. Theorems 3.1 and 3.2 improve and generalize Dien [4, Theorem 2], Ding [68, Theorems 3.1 and 3.2], Parida et al. [31, Theorems 3.1 and 3.9], and Yao [36, Theorem 3.1].

\section{Iterative method and convergence}

In this section, by using the auxiliary principle technique, we will give a general iterative method of approximate solutions of the generalized nonlinear mixed variational-like inequality problem (2.1) and convergence analysis of the iterative method. 
Let $D$ be a nonempty convex subset of a reflexive Banach space $E$ with dual space $E^{*}$. Let $N: E^{*} \times E^{*} \rightarrow E^{*}, T, A, B, C: D \rightarrow E^{*}$ be mappings. Suppose that $\eta: D \times D \rightarrow E$ is a mapping such that $\eta(v, u)=\eta(v, z)+\eta(z, u)$ for all $v, u, z \in D$. We consider an auxiliary differentiable convex functional $K: D \rightarrow(-\infty,+\infty]$ and a positive number $\rho>0$. For given $w^{*} \in D$ and $u^{*} \in D$, we introduce the following auxiliary minimizing problem:

$$
\begin{aligned}
\min _{w \in D}\{ & K(w)+\rho\left\langle N\left(T u^{*}, A u^{*}\right)-M\left(B u^{*}, C u^{*}\right)+w^{*}, \eta\left(w, u^{*}\right)\right\rangle \\
& \left.-\left\langle K^{\prime}\left(u^{*}\right), w\right\rangle+\rho b\left(u^{*}, w\right)\right\} .
\end{aligned}
$$

If the function $w \mapsto\left\langle N\left(T u^{*}, A u^{*}\right)-M\left(B u^{*}, C u^{*}\right)+w^{*}, \eta\left(w, u^{*}\right)\right\rangle$ is convex, then the solution $w$ of the auxiliary problem (4.1) can be characterized by the following auxiliary variational inequality problem:

$$
\begin{aligned}
\left\langle K^{\prime}(w)\right. & \left.-K^{\prime}\left(u^{*}\right), v-w\right\rangle \\
\geq & -\rho\left\langle N\left(T u^{*}, A u^{*}\right)-M\left(B u^{*}, C u^{*}\right)+w^{*}, \eta(v, u)\right\rangle \\
& +\rho b\left(u^{*}, w\right)-\rho b\left(u^{*}, v\right), \quad \forall v \in D .
\end{aligned}
$$

We note that if $w=u^{*}$, then $u^{*}$ is a solution of the generalized nonlinear mixed variational-like inequality problem (2.1). Based on these observations, we suggest the following iterative method for computing the approximate solutions of the problem (2.1).

Algorithm 4.1. (a) At $n=0$, start with some initial $u_{0}$.

(b) At step $n$, solve the auxiliary minimizing problem (4.1) or the auxiliary variational inequality problem (4.2) with $u^{*}=u_{n}$. Let $u_{n+1}$ denote the solution of problem (4.1) or (4.2).

(c) If, for given $\varepsilon>0,\left\|u_{n+1}-u_{n}\right\| \leq \varepsilon$, stop. Otherwise, repeat (b).

We now consider the convergence of Algorithm 4.1.

TheOREM 4.2. Let $D$ be a nonempty convex subset of a reflexive Banach space $E$ with dual space $E^{*}$. Let $M, N: E^{*} \times E^{*} \rightarrow E^{*}, T, A, B, C: D \rightarrow E^{*}$ and $\eta: D \times D \rightarrow E$ be mappings. Let $w^{*} \in E^{*}$ be given let $b: D \times D \rightarrow(-\infty,+\infty]$ be a functional, and let $K: E \rightarrow(-\infty,+\infty]$ be a differentiable convex functional such that

(a) the mapping $w \mapsto N(T w, A w)$ is continuous from the weak topology to the strong topology, $N$ is $\eta$-cocoercive in the first argument with respect to $T$ with constant $\alpha>$ 0 , and $\eta$-strongly monotone in the second argument with respect to $A$ with constant $\xi>0$, and is Lipschitz continuous in the first and second arguments with constants $\sigma_{1}$ and $\sigma_{2}$, respectively,

(b) the mapping $w \mapsto M(B w, C w)$ is continuous from the weak topology to the strong topology, $M$ is $\eta$-generalized pseudocontractive in the first argument with respect to $B$ with constant $\beta>0$, and $\eta$-relaxed Lipschitz in the second argument with respect to $C$ with constants $\sigma>0$ and $\beta>\sigma$, and is Lipschitz continuous in the first and second arguments with constants $\sigma_{3}>0$ and $\sigma_{4}>0$, respectively,

(c) $A, B, C: D \rightarrow E^{*}$ are Lipschitz continuous with constants $\sigma_{A}, \sigma_{B}, \sigma_{C}$, respectively,

(d) $\eta$ is Lipschitz continuous with constant $\delta \geq 0$ such that 
10 Generalized nonlinear mixed variational-like inequalities

(1) for each $v \in D, u \mapsto \eta(u, v)$ is continuous from the weak topology to the weak topology;

(2) for all $u, v, z \in D, \eta(u, v)=\eta(u, z)+\eta(z, v)$;

(3) for each fixed $u, w \in D$, the function $v \mapsto\left\langle N(T u, A u)-M(B u, C u)+w^{*}\right.$, $\eta(w, v)\rangle$ is concave,

(e) $b$ satisfies conditions ( 1$)-(\mathrm{c} 4)$ and $\beta+\nu<\xi+\sigma$,

(f) the derivative $K^{\prime}$ of $K$ is strongly convex with constant $\mu>0$ and the mapping $w \mapsto$ $K^{\prime}(w)$ is continuous from the weak topology to the strong topology.

Then

(A) the generalized nonlinear mixed variational-like inequality problem has a unique solution (2.1) $\hat{u} \in D$;

(B) for each $\rho>0$, there exists a unique solution $u_{n+1} \in D$ of Problems (4.1) or (4.2) with $u_{n}$ substituted for $u^{*}$;

(C) if

$$
0<\rho<\frac{2 \alpha \mu(\xi+\sigma-\beta-\nu)}{\sigma_{1}^{2} \delta^{2}(\xi+\sigma-\beta-\nu)+\alpha\left(\sigma_{2} \delta \sigma_{A}+\nu+\sigma_{3} \delta \sigma_{B}+\sigma_{4} \delta \sigma_{C}\right)^{2}},
$$

then the sequence $\left\{u_{n}\right\}$ defined by Algorithm 4.1 strongly converges to $\hat{u}$.

Proof. (A) In view of conditions (a) and (b), we get that the mapping $u \mapsto N(T u, A u)$ is $\eta$ strongly monotone with constant $\xi>0$ and the mapping $u \mapsto M(B u, C u)$ is $\eta$-generalized pseudocontractive with constant $\beta-\sigma$, respectively. Hence the conditions (a) and (b) of Theorem 3.2 are satisfied. By (3) of (d), for each $u \in D$, the function $v \mapsto\langle(T u, A u)-$ $\left.M(B u, C u)+w^{*}, \eta(u, v)\right\rangle$ is concave, it follows from Remark 2.4 that condition (d) of Theorem 3.1 holds. Noting that condition (2) of (d) implies that $\eta(u, u)=0$ and $\eta(u, v)=$ $-\eta(v, u)$ for all $u, v \in D$, it is easy to check that all conditions of Theorem 3.2 are satisfied. By Theorem 3.2, the conclusion (A) holds.

(B) For each fixed $\rho>0$ and $u_{n} \in D$, define a functional $\varphi: D \times D \rightarrow[-\infty,+\infty]$ by

$$
\begin{aligned}
\varphi(v, w)= & \left\langle K^{\prime}\left(u_{n}\right)-K^{\prime}(w), v-w\right\rangle \\
& -\rho\left\langle N\left(T u_{n}, A u_{n}\right)-M\left(B u_{n}, C u_{n}\right)+w^{*}, \eta(v, w)\right\rangle \\
& +\rho b\left(u_{n}, w\right)-\rho b\left(u_{n}, v\right) .
\end{aligned}
$$

Since $w \mapsto K^{\prime}(w)$ is continuous from the weak topology to the strong topology, it is easy to show that the function $w \mapsto\left\langle K^{\prime}(w), w\right\rangle$ is weakly continuous on $D$ and for each $v \in D$, $w \mapsto \varphi(v, w)$ is weak lower semicontinuous. By using similar argument as in the proof of Theorem 3.1, it is easy to show that $\varphi$ satisfied all conditions of Lemma 2.5, and hence there exists $\widehat{w} \in D$ such that

$$
\begin{aligned}
\left\langle K^{\prime}(\widehat{w})\right. & \left.-K^{\prime}\left(u_{n}\right), v-\widehat{w}\right\rangle \\
\geq & -\rho\left\langle N\left(T u_{n}, A u_{n}\right)-M\left(B u_{n}, C u_{n}\right)+w^{*}, \eta(v, \widehat{w})\right\rangle \\
& +\rho b\left(u_{n}, \widehat{w}\right)-\rho b\left(u_{n}, v\right), \quad \forall v \in D .
\end{aligned}
$$


It is easy to show that $\widehat{w}$ is unique, and hence $u_{n+1}=\widehat{w}$ is a unique solution of the auxiliary variational inequality problem (4.2).

(C) Let $\hat{u}$ be the unique solution of the generalized nonlinear mixed variational-like inequality problem (2.1). Define a functional $\Lambda: D \rightarrow(-\infty,+\infty]$ by

$$
\Lambda(u)=K(\hat{u})-K(u)-\left\langle K^{\prime}(u), \hat{u}-u\right\rangle, \quad \forall u \in D .
$$

Since $K^{\prime}$ is strongly convex, it follows that

$$
\Lambda(u) \geq \frac{\mu}{2}\|u-\hat{u}\|^{2}
$$

Note that $\eta(v, u)=-\eta(u, v)$ for all $v, u \in D$. It follows from (4.5) with $u_{n+1}=\widehat{w}$ and $v=\hat{u}$ that

$$
\begin{aligned}
\Lambda\left(u_{n}\right)- & \Lambda\left(u_{n+1}\right) \\
= & K\left(u_{n+1}\right)-K\left(u_{n}\right)-\left\langle K^{\prime}\left(u_{n}\right), u_{n+1}-u_{n}\right\rangle+\left\langle K^{\prime}\left(u_{n+1}\right)-K^{\prime}\left(u_{n}\right), \hat{u}-u_{n+1}\right\rangle \\
\geq & \frac{\mu}{2}\left\|u_{n+1}-u_{n}\right\|^{2}+\rho\left\langle N\left(T u_{n}, A u_{n}\right)-M\left(B u_{n}, C u_{n}\right)+w^{*}, \eta\left(u_{n+1}, \hat{u}\right)\right\rangle \\
& +\rho b\left(u_{n}, u_{n+1}\right)-\rho b\left(u_{n}, \widehat{u}\right) \\
= & \frac{\mu}{2}\left\|u_{n+1}-u_{n}\right\|^{2} \\
& +\rho\left\langle N\left(T u_{n}, A u_{n}\right)-M\left(B u_{n}, C u_{n}\right)-(N(T \hat{u}, A \hat{u})-M(B \hat{u}, C \hat{u})), \eta\left(u_{n+1}, \hat{u}\right)\right\rangle \\
& +\rho\left\langle N(T \hat{u}, A \hat{u})-M(B \hat{u}, C \hat{u})+w^{*}, \eta\left(u_{n+1}, \hat{u}\right)\right\rangle \\
& +\rho b\left(u_{n}, u_{n+1}\right)-\rho b\left(u_{n}, \hat{u}\right) .
\end{aligned}
$$

Since $\hat{u}$ is a solution of the generalized nonlinear mixed variational-like inequality problem (2.1) and $u_{n+1} \in D$, it follows that

$$
\left\langle N(T \widehat{u}, A \hat{u})-M(B \hat{u}, C \hat{u})+w^{*}, \eta\left(u_{n+1}, \widehat{u}\right)\right\rangle+b\left(\widehat{u}, u_{n+1}\right)-b(\hat{u}, \widehat{u}) \geq 0,
$$

which implies that

$$
\Lambda\left(u_{n}\right)-\Lambda\left(u_{n+1}\right) \geq \frac{\mu}{2}\left\|u_{n}-u_{n+1}\right\|^{2}+Q
$$

where

$$
\begin{aligned}
Q= & \rho\left\langle N\left(T u_{n}, A u_{n}\right)-M\left(B u_{n}, C u_{n}\right)-(N(T \hat{u}, A \hat{u})-M(B \hat{u}, C \hat{u})), \eta\left(u_{n+1}, \hat{u}\right)\right\rangle \\
& -\rho\left[b\left(u_{n}-\hat{u}, \hat{u}-u_{n}\right)+b\left(u_{n}-\widehat{u}, u_{n}-u_{n+1}\right)\right] .
\end{aligned}
$$


12 Generalized nonlinear mixed variational-like inequalities

By the conditions (a)-(f), we deduce that

$$
\begin{aligned}
& Q=\rho\left\langle N\left(T u_{n}, A u_{n}\right)-N(T \hat{u}, A \hat{u}), \eta\left(u_{n+1}, u_{n}\right)\right\rangle \\
& -\rho\left\langle M\left(B u_{n}, C u_{n}\right)-M(B \hat{u}, C \hat{u}), \eta\left(u_{n+1}, u_{n}\right)\right\rangle \\
& +\rho\left\langle N\left(T u_{n}, A u_{n}\right)-N(T \hat{u}, A \hat{u}), \eta\left(u_{n}, \widehat{u}\right)\right\rangle \\
& -\rho\left\langle M\left(B u_{n}, C u_{n}\right)-M(B \hat{u}, C \hat{u}), \eta\left(u_{n}, \hat{u}\right)\right\rangle \\
& -\rho\left[b\left(u_{n}-\hat{u}, \hat{u}-u_{n}\right)+b\left(u_{n}-\hat{u}, u_{n}-u_{n+1}\right)\right] \\
& \geq \rho\left\langle N\left(T u_{n}, A u_{n}\right)-N\left(T \hat{u}, A u_{n}\right), \eta\left(u_{n}, \hat{u}\right)\right\rangle \\
& +\rho\left\langle N\left(T \hat{u}, A u_{n}\right)-N(T \hat{u}, A \hat{u}), \eta\left(u_{n}, \hat{u}\right)\right\rangle \\
& +\rho\left\langle N\left(T u_{n}, A u_{n}\right)-N\left(T \hat{u}, A u_{n}\right), \eta\left(u_{n+1}, u_{n}\right)\right\rangle \\
& +\rho\left\langle N\left(T \hat{u}, A u_{n}\right)-N(T \hat{u}, A \hat{u}), \eta\left(u_{n+1}, u_{n}\right)\right\rangle \\
& -\rho\left\langle M\left(B u_{n}, C u_{n}\right)-M\left(B \hat{u}, C u_{n}\right), \eta\left(u_{n}, \hat{u}\right)\right\rangle \\
& -\rho\left\langle M\left(B \widehat{u}, C u_{n}\right)-M(B \widehat{u}, C \hat{u}), \eta\left(u_{n}, \widehat{u}\right)\right\rangle \\
& -\rho\left\langle M\left(B u_{n}, C u_{n}\right)-M\left(B \hat{u}, C u_{n}\right), \eta\left(u_{n+1}, u_{n}\right)\right\rangle \\
& -\rho\left\langle M\left(B \hat{u}, C u_{n}\right)-M(B \hat{u}, C \hat{u}), \eta\left(u_{n+1}, u_{n}\right)\right\rangle \\
& -\rho v\left[\left\|u_{n}-\hat{u}\right\|^{2}+\left\|u_{n}-\hat{u}\right\|\left\|u_{n}-u_{n+1}\right\|\right] \\
& \geq \rho\left[\alpha\left\|T u_{n}-T \hat{u}\right\|^{2}-\sigma_{1} \delta\left\|T u_{n}-T \hat{u}\right\|\left\|u_{n+1}-u_{n}\right\|\right] \\
& +\rho \xi\left\|u_{n}-\hat{u}\right\|^{2}-\rho \sigma_{2} \delta \sigma_{A}\left\|u_{n}-\hat{u}\right\|\left\|u_{n+1}-u_{n}\right\|-\rho \nu\left\|u_{n}-\hat{u}\right\|^{2} \\
& -\rho \nu\left\|u_{n}-\hat{u}\right\|\left\|u_{n}-u_{n+1}\right\|-\rho \beta\left\|u_{n}-\hat{u}\right\|^{2}+\rho \sigma\left\|u_{n}-\hat{u}\right\|^{2} \\
& -\rho \delta \sigma_{3} \sigma_{B}\left\|u_{n}-\hat{u}\right\||| u_{n+1}-u_{n}\left\|-\rho \delta \sigma_{4} \sigma_{C}\right\| u_{n}-\hat{u}\|\| u_{n+1}-u_{n} \| \\
& \geq \rho\left[-\frac{\sigma_{1}^{2} \delta^{2}}{4 \alpha}\right]\left\|u_{n+1}-u_{n}\right\|^{2} \\
& -\rho\left(\sigma_{2} \delta \sigma_{A}+\nu+\delta \sigma_{3} \sigma_{B}+\delta \sigma_{4} \sigma_{C}\right)\left\|u_{n}-\hat{u}\right\|\left\|u_{n+1}-u_{n}\right\| \\
& +\rho(\xi+\sigma-\beta-\nu)\left\|u_{n}-\hat{u}\right\|^{2} \text {. }
\end{aligned}
$$

Therefore, we have

$$
\begin{aligned}
\Lambda\left(u_{n}\right)- & \Lambda\left(u_{n+1}\right) \\
\geq & \frac{1}{2}\left(\mu-\frac{\rho \sigma_{1}^{2} \delta^{2}}{2 \alpha}\right)\left\|u_{n+1}-u_{n}\right\|^{2} \\
& -\rho\left(\sigma_{2} \delta \sigma_{A}+\nu+\delta \sigma_{3} \sigma_{B}+\delta \sigma_{4} \sigma_{C}\right)\left\|u_{n}-\hat{u}\right\|\left\|u_{n+1}-u_{n}\right\| \\
& +\rho(\xi+\sigma-\beta-\nu)\left\|u_{n}-\hat{u}\right\|^{2} \\
\geq & {\left[\rho(\xi+\sigma-\beta-\nu)-\frac{\rho^{2}\left(\sigma_{2} \delta \sigma_{A}+\nu+\delta \sigma_{3} \sigma_{B}+\delta \sigma_{4} \sigma_{C}\right)^{2}}{2\left(\mu-\rho \sigma_{1}^{2} \delta^{2} / 2 \alpha\right)}\right]\left\|u_{n}-\hat{u}\right\|^{2} . }
\end{aligned}
$$


The conditions (4.3), (4.7), and (4.13) show that the sequence $\left\{\Lambda\left(u_{n}\right)\right\}$ is non-negative and decreasing. Hence it converges to some number and the sequence $\left\{u_{n}\right\}$ converges strongly to $\hat{u}$ as $n \rightarrow \infty$. This completes the proof.

Remark 4.3. Theorem 4.2 improves and generalizes Ding [7, Theorem 4.1], Ding [8, Theorem 4.1 and Corollary 4.1], and Cohen [3, Theorem 2.2].

\section{Acknowledgments}

The authors would like to express their thanks to the referees for helpful comments and suggestions. This work was supported by the Tianyuan Grant from the National Science Foundation of China (no. 10426008) and the Science Research Foundation of Educational Department of Liaoning Province (2006).

\section{References}

[1] Q. H. Ansari and J. C. Yao, Iterative schemes for solving mixed variational-like inequalities, Journal of Optimization Theory and Applications 108 (2001), no. 3, 527-541.

[2] X. H. Chen and Y. F. Liu, A generalized nonlinear variational-like inequality in reflexive Banach spaces, Journal of Nanjing University. Mathematical Biquarterly 18 (2001), no. 1, 96-103.

[3] G. Cohen, Auxiliary problem principle extended to variational inequalities, Journal of Optimization Theory and Applications 59 (1988), no. 2, 325-333.

[4] N. H. Dien, Some remarks on variational-like and quasivariational-like inequalities, Bulletin of the Australian Mathematical Society 46 (1992), no. 2, 335-342.

[5] X. P. Ding, General algorithm of solutions for nonlinear variational inequalities in Banach space, Computers \& Mathematics with Applications 34 (1997), no. 9, 131-137.

[6] __ Algorithm of solutions for mixed-nonlinear variational-like inequalities in reflexive Banach space, Applied Mathematics and Mechanics 19 (1998), no. 6, 521-529.

[7] __ General algorithm for nonlinear variational-like inequalities in reflexive Banach spaces, Indian Journal of Pure and Applied Mathematics 29 (1998), no. 2, 109-120.

[8] __ Existence and algorithm of solutions for nonlinear mixed variational-like inequalities in Banach spaces, Journal of Computational and Applied Mathematics 157 (2003), no. 2, 419-434.

[9] X. P. Ding and K.-K. Tan, A minimax inequality with applications to existence of equilibrium point and fixed point theorems, Colloquium Mathematicum 63 (1992), no. 2, 233-247.

[10] X. P. Ding and E. Tarafdar, Existence and uniqueness of solutions for a general nonlinear variational inequality, Applied Mathematics Letters 8 (1995), no. 1, 31-36.

[11] R. Glowinski, J.-L. Lions, and R. Trémolières, Numerical Analysis of Variational Inequalities, Studies in Mathematics and Its Applications, vol. 8, North-Holland, Amsterdam, 1981.

[12] N.-j. Huang and C.-x. Deng, Auxiliary principle and iterative algorithms for generalized set-valued strongly nonlinear mixed variational-like inequalities, Journal of Mathematical Analysis and Applications 256 (2001), no. 2, 345-359.

[13] S. Karamardian, The nonlinear complementarity problem with applications. II, Journal of Optimization Theory and Applications 4 (1969), 167-181.

[14] D. Kinderlehrer and G. Stampacchia, An Introduction to Variational Inequalities and Their Applications, Pure and Applied Mathematics, vol. 88, Academic Press, New York, 1980. 
[15] Z. Liu, L. Debnath, S. M. Kang, and J. S. Ume, Completely generalized multivalued nonlinear quasi-variational inclusions, International Journal of Mathematics and Mathematical Sciences 30 (2002), no. 10, 593-604.

[16] On the generalized nonlinear quasivariational inclusions, Acta Mathematica et Informatica Universitatis Ostraviensis 11 (2003), no. 1, 81-90.

[17] Sensitivity analysis for parametric completely generalized nonlinear implicit quasivariational inclusions, Journal of Mathematical Analysis and Applications 277 (2003), no. 1, 142-154.

[18]__ Generalized mixed quasivariational inclusions and generalized mixed resolvent equations for fuzzy mappings, Applied Mathematics and Computation 149 (2004), no. 3, 879-891.

[19] Z. Liu and S. M. Kang, Comments on the papers involving variational and quasi-variational inequalities for fuzzy mappings, Mathematical Sciences Research Journal 7 (2003), no. 10, 394-399.

[20] _ Generalized multivalued nonlinear quasivariational inclusions, Mathematische Nachrichten 253 (2003), no. 1, 45-54.

[21]__ Convergence and stability of perturbed three-step iterative algorithm for completely generalized nonlinear quasivariational inequalities, Applied Mathematics and Computation 149 (2004), no. 1, 245-258.

[22] Z. Liu, S. M. Kang, and J. S. Ume, On general variational inclusions with noncompact valued mappings, Advances in Nonlinear Variational Inequalities 5 (2002), no. 2, 11-25.

[23] Completely generalized multivalued strongly quasivariational inequalities, Publicationes Mathematicae Debrecen 62 (2003), no. 1-2, 187-204.

[24] _ Generalized variational inclusions for fuzzy mappings, Advances in Nonlinear Variational Inequalities 6 (2003), no. 1, 31-40.

[25] The solvability of a class of quasivariational inequalities, Advances in Nonlinear Variational Inequalities 6 (2003), no. 2, 69-78.

[26] Y.-p. Liu and Y.-y. Tang, Generalized strongly nonlinear mixed variational-like inequalities in reflexive Banach spaces, Journal of Sichuan University 41 (2004), no. 3, 489-492.

[27] Z. Liu, J. S. Ume, and S. M. Kang, General strongly nonlinear quasivariational inequalities with relaxed Lipschitz and relaxed monotone mappings, Journal of Optimization Theory and Applications 114 (2002), no. 3, 639-656.

[28] _ Resolvent equations technique for general variational inclusions, Proceedings of the Japan Academy. Series A 78 (2002), no. 10, 188-193.

[29] - Nonlinear variational inequalities on reflexive Banach spaces and topological vector spaces, International Journal of Mathematics and Mathematical Sciences 2003 (2003), no. 4, 199-207.

[30] Completely generalized quasivariational inequalities, Advances in Nonlinear Variational Inequalities 7 (2004), no. 1, 35-46.

[31] J. Parida, M. Sahoo, and A. Kumar, A variational-like inequality problem, Bulletin of the Australian Mathematical Society 39 (1989), no. 2, 225-231.

[32] A. H. Siddiqi, Q. H. Ansari, and R. Ahmad, On generalized variational-like inequalities, Indian Journal of Pure and Applied Mathematics 26 (1995), no. 12, 1135-1141.

[33] A. H. Siddiqi, A. Khaliq, and Q. H. Ansari, On variational-like inequalities, Annales des Sciences Mathématiques du Québec 18 (1994), no. 1, 95-104.

[34] P. Tseng, Further applications of a splitting algorithm to decomposition in variational inequalities and convex programming, Mathematical Programming. Series B 48 (1990), no. 2, 249-263.

[35] R. U. Verma, On generalized variational inequalities involving relaxed Lipschitz and relaxed monotone operators, Journal of Mathematical Analysis and Applications 213 (1997), no. 1, 387-392. 
[36] J. C. Yao, Existence of generalized variational inequalities, Operations Research Letters 15 (1994), no. $1,35-40$.

[37] Y. Zhao, Z. Liu, and S. M. Kang, On the generalized set-valued mixed variational inequalities, Communications of the Korean Mathematical Society 18 (2003), no. 3, 459-468.

Yali Zhao: Department of Applied Mathematics, Dalian University of Technology,

Dalian, Liaoning 116024, China

E-mail address: yalizhao2000@yahoo.com.cn

Zunquan Xia: Department of Applied Mathematics, Dalian University of Technology, Dalian, Liaoning 116024, China

E-mail address: zqxiazhh@dlut.edu.cn

Zeqing Liu: Department of Mathematics, Liaoning Normal University, P.O. Box 200, Dalian, Liaoning 116029, China

E-mail address: zeqingliu@dl.cn

Shin Min Kang: Department of Mathematics and Research Institute of Natural Science,

Gyeongsang National University, Jinju 660-701, Korea

E-mail address: smkang@nongae.gsnu.ac.kr 


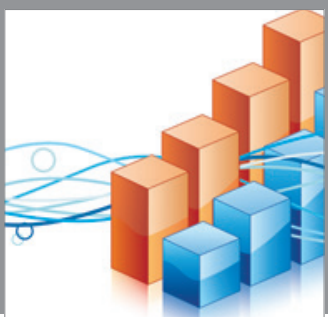

Advances in

Operations Research

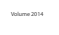

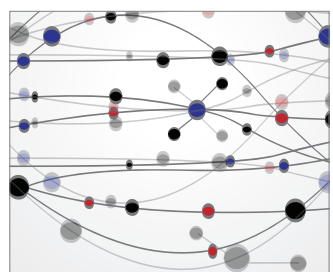

\section{The Scientific} World Journal
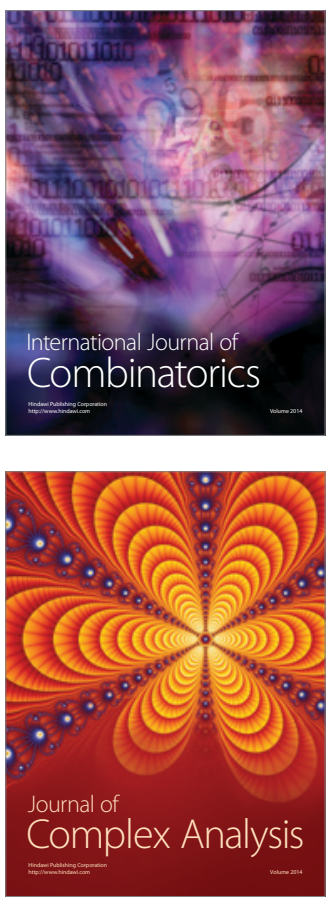

International Journal of

Mathematics and

Mathematical

Sciences
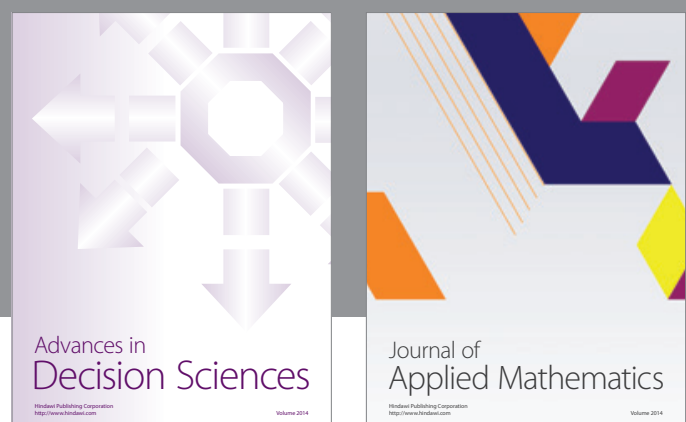

Journal of

Applied Mathematics
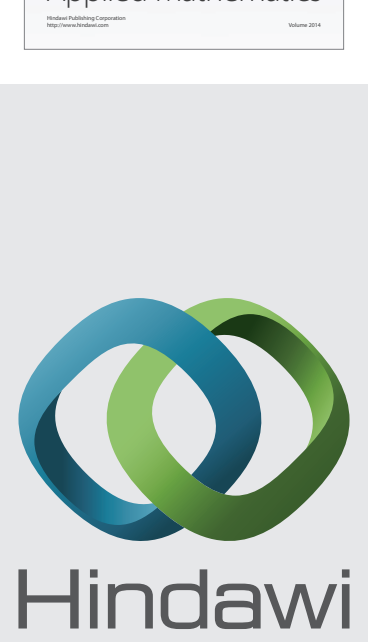

Submit your manuscripts at http://www.hindawi.com
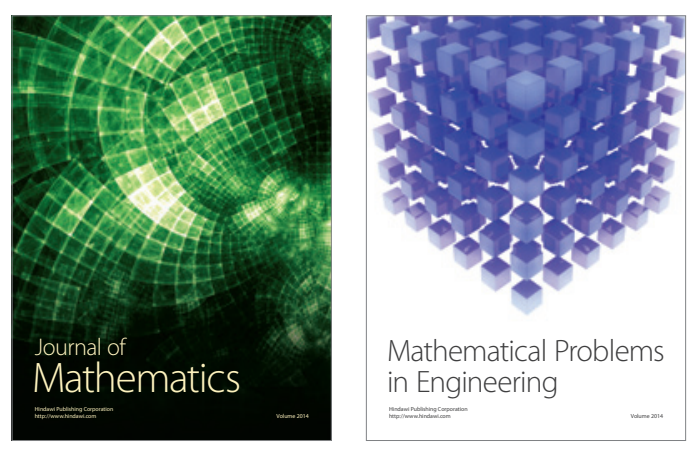

Mathematical Problems in Engineering
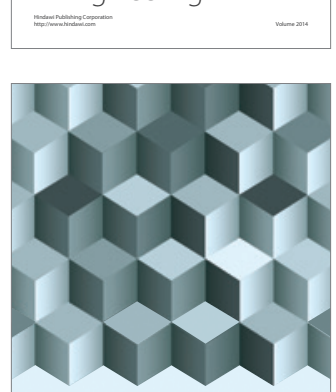

Journal of

Function Spaces
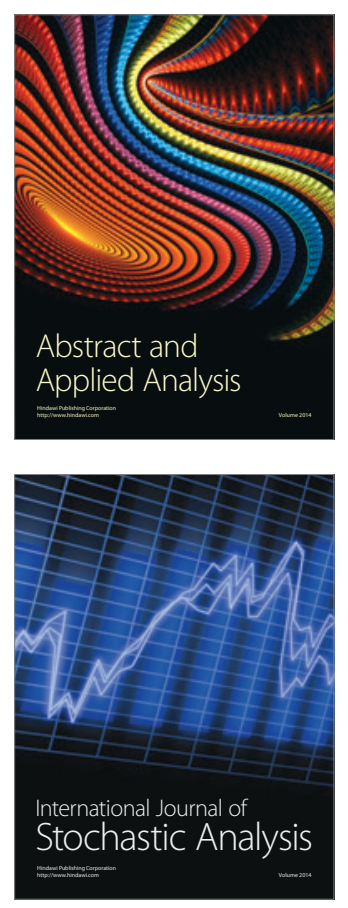

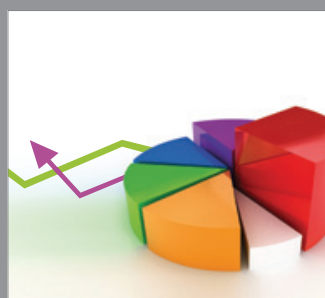

ournal of

Probability and Statistics

Promensencen
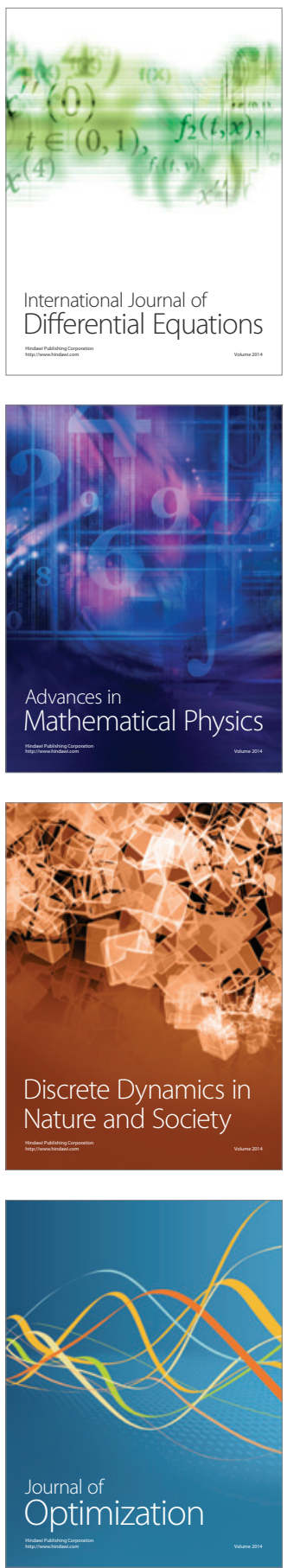